\title{
Infinitely Many Solutions for Perturbed Hemivariational Inequalities
}

\author{
Giuseppina D'Aguì', 2 and Giovanni Molica Bisci ${ }^{3}$ \\ ${ }^{1}$ DIMET, Faculty of Engineering, University of Reggio Calabria, 89125 Reggio Calabria, Italy \\ ${ }^{2}$ DiSIA, Faculty of Engineering, University of Messina, 98122 Messina, Italy \\ ${ }^{3}$ Department P.A.U., Architecture Faculty, University of Reggio Calabria, 89100 Reggio Calabria, Italy
}

Correspondence should be addressed to Giovanni Molica Bisci, gmolica@unirc.it

Received 8 September 2010; Accepted 28 November 2010

Academic Editor: Raul F. Manasevich

Copyright (c) 2010 G. D’Aguì and G. Molica Bisci. This is an open access article distributed under the Creative Commons Attribution License, which permits unrestricted use, distribution, and reproduction in any medium, provided the original work is properly cited.

We deal with a perturbed eigenvalue Dirichlet-type problem for an elliptic hemivariational inequality involving the $p$-Laplacian. We show that an appropriate oscillating behaviour of the nonlinear part, even under small perturbations, ensures the existence of infinitely many solutions. The main tool in order to obtain our abstract results is a recent critical-point theorem for nonsmooth functionals.

\section{Introduction}

Hemivariational inequalities appear in the mathematical modeling of several complicated mechanical and engineering problems, whose relevant energy functionals are neither convex nor smooth. For instance, this is the case of non-monotone multivalued interface laws or constitutive relations that occur in certain contact and friction processes, as well as of phenomena related to large displacements and deformations expressed by nonlinear straindisplacement laws.

The theory of hemivariational inequalities can be viewed as a new field of nonsmooth mechanics since the main ingredient used in the study of these inequalities is the notion of the Clarke subdifferential of a locally Lipschitz continuous functional. The mathematical theory hemivariational inequalities, as well as their applications in mechanics, engineering or economics were introduced and developed by Panagiotopoulos [1, 2].

For a treatment of this topics, we refer the reader to the monographs by Naniewicz and Panagiotopoulos [3], Motreanu and Panagiotopoulos [4], Motreanu and Rădulescu [5, 6], 
and Rădulescu [7, 8]. Moreover, very recently, several inequalities problems have been studied; see, for instance, the papers [9-14].

Our work is inspired by the very nice and seminal papers of Marano and Motranu $[13,15]$. In particular, by following [13], where the authors stated the existence of infinitely many solutions of a Neumann-type problem for an elliptic variational-hemivariational inequality, in this paper we treat a Dirichlet-type problem for an elliptic hemivariational inequality driven by the $p$-Laplacian, by using a different technique (see Remark 3.2).

Precisely, let $\Omega$ be a nonempty, bounded, open subset of the real Euclidean $N$-dimensional space $\left(\mathbb{R}^{N},|\cdot|\right), N \geq 1$, with smooth boundary $\left.\partial \Omega, p \in\right] N,+\infty[$, and let $f, g: \mathbb{R} \rightarrow \mathbb{R}$ be two locally essentially bounded functions. Set $\alpha, \beta \in L^{1}(\Omega)$ such that $\min \{\alpha(x), \beta(x)\} \geq 0$, almost everywhere in $\Omega$ and, finally, $\lambda, \mu \in \mathbb{R}$ with $\lambda>0$ and $\mu \geq 0$. The main purpose is to study the following elliptic hemivariational inequality problem, say $\left(P_{\lambda, \mu}^{f, g}\right)$.

$$
\begin{aligned}
& \text { Find } u \in W_{0}^{1, p}(\Omega) \text { fulfilling } \\
& -\int_{\Omega}|\nabla u(x)|^{p-2} \nabla u(x) \cdot \nabla(v(x)-u(x)) d x \\
& \quad \leq \lambda \int_{\Omega} \alpha(x) F^{\circ}(u(x) ;(v(x)-u(x))) d x+\mu \int_{\Omega} \beta(x) G^{\circ}(u(x) ;(v(x)-u(x))) d x,
\end{aligned}
$$

for every $v \in W_{0}^{1, p}(\Omega)$, where $F^{\circ}$ and $G^{\circ}$ are the generalized directional derivatives of the locally Lipschitz continuous functions

$$
F(\xi):=\int_{0}^{\xi} f(t) d t, \quad G(\xi):=\int_{0}^{\xi} g(t) d t
$$

for every $\xi \in \mathbb{R}$.

Here, by using a recent abstract critical-point result (see Theorem 2.2 below), under some hypotheses on the behavior of the potential of the nonlinear term $f$ at infinity, we show the existence of a precise interval of parameters $\Lambda$ such that, for each $\lambda \in \Lambda$, and every locally essentially bounded function $g$ that satisfies certain conditions at infinity, the perturbed problem $\left(P_{\lambda, \mu}^{f, g}\right)$ admits a sequence of solutions which are unbounded in the Sobolev space $W_{0}^{1, p}(\Omega)$; see Theorem 3.1.

Further, replacing the conditions at infinity of the potential of $f$ and of the perturbation term $g$, by a similar one at zero, the same results hold and, in addition, the sequence of pairwise distinct solutions uniformly converges to zero; see Theorem 3.6.

If $f, g \in C^{0}(\mathbb{R})$, as pointed out in Remark 3.5 below, a function $u \in W_{0}^{1, p}(\Omega)$ solves $\left(P_{\lambda, \mu}^{f, g}\right)$ if and only if it is a weak solution of the Dirichlet problem

$$
\begin{gathered}
\Delta_{p} u=\lambda \alpha(x) f(u)+\mu \beta(x) g(u) \text { in } \Omega, \\
\left.u\right|_{\partial \Omega}=0,
\end{gathered}
$$$$
\left(D_{\lambda, \mu}^{f, g}\right)
$$

where $\Delta_{p}=\operatorname{div}\left(|\nabla u|^{p-2} \nabla u\right)$ is the $p$-Laplacian operator. 
The existence of infinitely many solutions for some elliptic Dirichlet problems has received a great deal of interest in recent years; see, for instance, the papers [16-20]. Moreover, very recently, existence results for problems involving a perturbation term have been studied in [21-23].

Here, we present a special case of our result.

Theorem 1.1. Let $f: \mathbb{R} \rightarrow \mathbb{R}$ be a nonpositive continuous function with potential $F(\xi):=\int_{0}^{\xi} f(t) d t$, for every $\xi \in \mathbb{R}$. Assume that the following condition holds:

$$
\left(f_{2}^{\prime \prime}\right) \liminf _{\xi \rightarrow+\infty}\left((-F(\xi)) / \xi^{2}\right)<(1 / 4) \lim \sup _{\xi \rightarrow+\infty}\left((-F(\xi)) / \xi^{2}\right) .
$$

Then, for each

$$
\lambda \in \Lambda:=] \frac{8}{\limsup _{\xi \rightarrow+\infty}\left((-F(\xi)) / \xi^{2}\right)}, \frac{2}{\liminf _{\xi \rightarrow+\infty}\left((-F(\xi)) / \xi^{2}\right)}[
$$

for every nonpositive continuous function $g: \mathbb{R} \rightarrow \mathbb{R}$ such that

$$
\left(g_{1}^{\prime}\right) G_{\infty}^{\star}:=\lim _{\xi \rightarrow+\infty}\left(\int_{0}^{\xi} g(t) d t / \xi^{2}\right)>-\infty,
$$

for every $\mu \in\left[0, \widehat{\mu}_{g, \lambda}[\right.$, where

$$
\widehat{\mu}_{g, \lambda}:=\frac{1}{G_{\infty}^{\star}}\left(2-\lambda \liminf _{\xi \rightarrow+\infty} \frac{(-F(\xi))}{\xi^{2}}\right)
$$

the following problem

$$
\begin{gathered}
\left.u^{\prime \prime}=\lambda f(u)+\mu g(u) \text { in }\right] 0,1[ \\
u(0)=u(1)=0
\end{gathered}
$$

admits a sequence of pairwise distinct positive classical solutions.

The present paper is arranged as follows. In Section 2, we recall some basic definitions and preliminary results, while Section 3 is devoted to the existence of infinitely many solutions for the eigenvalue problem $\left(P_{\lambda, \mu}^{f, g}\right)$. Finally, in Section 4 , we give concrete examples of application of our abstract results.

\section{Preliminaries}

Let $(X,\|\cdot\|)$ be a real Banach space. We denote by $X^{*}$ the dual space of $X$, while $\langle\cdot, \cdot\rangle$ stands for the duality pairing between $X^{*}$ and $X$. A function $h: X \rightarrow \mathbb{R}$ is called locally Lipschitz continuous when to every $x \in X$, there correspond a neighborhood $V_{x}$ of $x$ and a constant $L_{x} \geq 0$ such that

$$
|h(z)-h(w)| \leq L_{x}\|z-w\|, \quad \forall z, w \in V_{x} .
$$


If $x, z \in X$, we write $h^{\circ}(x ; z)$ for the generalized directional derivative of $h$ at the point $x$ along the direction $z$, that is,

$$
h^{\circ}(x ; z):=\limsup _{w \rightarrow x, t \rightarrow 0^{+}} \frac{h(w+t z)-h(w)}{t} .
$$

The generalized gradient of the function $h$ in $x$, denoted by $\partial h(x)$, is the set

$$
\partial h(x):=\left\{x^{*} \in X^{*}:\left\langle x^{*}, z\right\rangle \leq h^{\circ}(x ; z), \forall z \in X\right\} .
$$

Two meaningful properties of generalized directional derivatives are collected in the proposition below. Their proofs and a thorough treatment of this topic can be found in [24]; let us mention [25] too.

Proposition 2.1. Let $h: X \rightarrow \mathbb{R}$ be locally Lipschitz continuous functional. Then, $h^{\circ}: X \times X \rightarrow \mathbb{R}$ is upper semicontinuous and for all $\lambda \geq 0, x, z \in X$, one has

$$
(\lambda h)^{\circ}(x ; z)=\lambda h^{\circ}(x ; z)
$$

Moreover, if $h_{1}, h_{2}: X \rightarrow \mathbb{R}$ are locally Lipschitz continuous functional, then

$$
\left(h_{1}+h_{2}\right)^{\circ}(x, z) \leq h_{1}^{\circ}(x, z)+h_{2}^{\circ}(x, z), \quad \forall x, z \in X
$$

When a nonsmooth functional, $g: X \rightarrow]-\infty,+\infty]$, is expressed as a sum of a locally Lipschitz continuous function, $h: X \rightarrow \mathbb{R}$, and a convex, proper, and lower semicontinuous function, $j: X \rightarrow]-\infty,+\infty$ ], that is, $g:=h+j$, a (generalized) critical point of $g$ is every $x \in X$ such that

$$
h^{\circ}(x ; z-x)+j(z)-j(x) \geq 0,
$$

for all $z \in X$; see [4, Chapter 3].

If $j \equiv 0$, then it clearly signifies $0 \in \partial h(x)$, namely, $x$ is a critical point of $h$ according to [25, Definition 2.1].

From now, assume that $X$ is a reflexive real Banach space, $\Phi: X \rightarrow \mathbb{R}$ is a sequentially weakly lower semicontinuous functional, $\Upsilon: X \rightarrow \mathbb{R}$ is a sequentially weakly upper semicontinuous functional, $\lambda$ is a positive real parameter, $j: X \rightarrow]-\infty,+\infty]$ is a convex, proper and lower semicontinuous functional, and $D(j)$ is the effective domain of $j$.

Write

$$
\Psi:=\Upsilon-j, \quad J_{\lambda}:=\Phi-\lambda \Psi=(\Phi-\lambda \Upsilon)+\lambda j
$$

We also assume that $\Phi$ is coercive and

$$
D(j) \cap \Phi^{-1}(]-\infty, r[) \neq \emptyset,
$$


for all $r>\inf _{X} \Phi$. Moreover, from (2.8) and provided $r>\inf _{X} \Phi$, we can define

$$
\begin{gathered}
\varphi(r)=\inf _{u \in \Phi^{-1}(]-\infty, r[)} \frac{\left(\sup _{v \in \Phi^{-1}(]-\infty, r[)} \Psi(v)\right)-\Psi(u)}{r-\Phi(u)}, \\
r:=\liminf _{r \rightarrow+\infty} \varphi(r), \quad \delta:=\liminf _{\left.r \rightarrow \inf _{x} \Phi\right)^{+}} \varphi(r) .
\end{gathered}
$$

If $\Phi$ and $\Upsilon$ are locally Lipschitz continuous functionals, in [26] the following result is proved, which is a more precise version of [13, Theorem 1.1] (see also [27]).

Theorem 2.2. Under the above assumptions on $X, \Phi$, and $\Psi$, one has

(a) If $\gamma<+\infty$, then, for each $\lambda \in] 0,1 / \gamma[$, the following alternative holds:

either

$\left(\mathrm{a}_{1}\right) J_{\lambda}$ possesses a global minimum,

or

( $\left.\mathrm{a}_{2}\right)$ there is a sequence $\left\{u_{n}\right\}$ of critical points (local minima) of $J_{\mathcal{\lambda}}$ such that $\lim _{n \rightarrow \infty} \Phi\left(u_{n}\right)=+\infty$.

(b) If $\delta<+\infty$, then, for each $\lambda \in] 0,1 / \delta[$, the following alternative holds:

either

$\left(\mathrm{b}_{1}\right)$ there is a global minimum of $\Phi$ which is a local minimum of $J_{\lambda}$,

or

$\left(b_{2}\right)$ there is a sequence $\left\{u_{n}\right\}$ of pairwise distinct critical points (local minima) of $J_{\lambda}$, with $\lim _{n \rightarrow \infty} \Phi\left(u_{n}\right)=\inf _{X} \Phi$, which weakly converges to a global minimum of $\Phi$.

We recall here some basic definitions and notations. As usual, $W_{0}^{1, p}(\Omega)$ is the closure of $C_{0}^{\infty}(\Omega)$ with respect to the norm

$$
\|u\|=\left(\int_{\Omega}|\nabla u(x)|^{p} d x\right)^{1 / p}
$$

We are interested in the existence of infinitely many solutions for problem $\left(P_{\lambda, \mu}^{f, g}\right)$. The main objective is to use the abstract Theorem 2.2.

Put

$$
k:=\sup \left\{\frac{\max _{x \in \bar{\Omega}}|u(x)|}{\|u\|}: u \in W_{0}^{1, p}(\Omega), u \neq 0\right\}
$$


Since $p>N$, one has $k<+\infty$. For our goal, it is enough to know an explicit upper bound for the constant $k$. Denoting by $\Gamma$ the Gamma function defined by

$$
\Gamma(t):=\int_{0}^{+\infty} z^{t-1} e^{-z} d z, \quad \forall t>0
$$

In this connection, it is well known (see [28, formula (6b)]) that if we set

$$
m:=\frac{N^{-1 / p}}{\sqrt{\pi}}\left[\Gamma\left(1+\frac{N}{2}\right)\right]^{1 / N}\left(\frac{p-1}{p-N}\right)^{1-1 / p} \operatorname{meas}(\Omega)^{1 / N-1 / p},
$$

where "meas $(\Omega)$ " is the Lebesgue measure of $\Omega$, one has $k \leq m$ (equality occurs when $\Omega$ is a ball). Then,

$$
\|u\|_{\infty} \leq m\|u\|
$$

for every $u \in W_{0}^{1, p}(\Omega)$. Define

$$
\sigma(N, p):=\inf _{\eta \in] 0,1[} \frac{1-\eta^{N}}{\eta^{N}(1-\eta)^{p}}
$$

and consider $\bar{\eta} \in] 0,1\left[\right.$ such that $\sigma(N, p)=\left(1-\bar{\eta}^{N}\right) /\left(\bar{\eta}^{N}(1-\bar{\eta})^{p}\right)$. Moreover, let

$$
\tau:=\sup _{x \in \Omega} \operatorname{dist}(x, \partial \Omega),
$$

where "dist $(x, \partial \Omega)$ " denotes the usual Euclidean distance from $x \in \Omega$ to the boundary $\partial \Omega$. Simple calculations show that there is $x_{0} \in \Omega$ such that $B\left(x_{0}, \tau\right) \subseteq \Omega$. Further, put

$$
\mathcal{\kappa}:=\left(\frac{\tau^{p}}{m^{p}\|\alpha\|_{L^{1}(\Omega)} \sigma(N, p)}\right) \frac{\|\alpha\|_{L^{1}\left(B\left(x_{0}, \bar{\eta} \tau\right)\right)}}{\bar{\eta}^{N} \omega_{\tau}}
$$

where $m$ is given by (2.13). We also denote by

$$
\omega_{\tau}:=\tau^{N} \frac{\pi^{N / 2}}{\Gamma(1+N / 2)}
$$

the measure of the $N$-dimensional ball of radius $\tau$. Finally, let

$$
\begin{gathered}
A:=\liminf _{\xi \rightarrow+\infty} \frac{\max _{|t| \leq \xi}(-F(t))}{\xi^{p}}, \quad B:=\limsup _{\xi \rightarrow+\infty} \frac{(-F(\xi))}{\xi^{p}} \\
\lambda_{1}:=\frac{\omega_{\tau} \bar{\eta}^{N} \sigma(N, p)}{p \tau^{p}\|\alpha\|_{L^{1}\left(B\left(x_{0}, \bar{\eta} \tau\right)\right)} B^{\prime}}, \quad \lambda_{2}:=\frac{1}{p m^{p}\|\alpha\|_{L^{1}(\Omega)} A} .
\end{gathered}
$$




\section{Main Results}

In this section, we present our main existence result for the hemivariational inequality problem $\left(P_{\lambda, \mu}^{f, g}\right)$.

Theorem 3.1. Let $f: \mathbb{R} \rightarrow \mathbb{R}$ be a locally essentially bounded function and put $F(\xi):=\int_{0}^{\xi} f(t) d t$, for every $\xi \in \mathbb{R}$. Assume that

$\left(f_{1}\right) \sup _{\xi \geq 0} F(\xi)=0$

$\left(f_{2}\right)$ and that

$$
\liminf _{\xi \rightarrow+\infty} \frac{\max _{|t| \leq \xi \xi}(-F(t))}{\xi^{p}}<\kappa \limsup _{\xi \rightarrow+\infty} \frac{(-F(\xi))}{\xi^{p}}
$$

where $\kappa$ is given by (2.17).

Then, for every $\lambda \in] \lambda_{1}, \lambda_{2}[$, for every locally essentially bounded function $g: \mathbb{R} \rightarrow \mathbb{R}$ whose potential $G(\xi):=\int_{0}^{\xi} g(t) d t$ for every $\xi \in \mathbb{R}$, satisfies

$$
\begin{aligned}
& \left(g_{1}\right) \sup _{\xi \geq 0} G(\xi)=0, \\
& \left(g_{2}\right) G_{\infty}:=\lim _{\xi \rightarrow+\infty}\left(\max _{|t| \leq \xi}(-G(t)) / \xi^{p}\right)<+\infty,
\end{aligned}
$$

and for every $\mu \in\left[0, \mu_{g, \lambda}[\right.$, where

$$
\mu_{g, \lambda}:=\frac{1}{\|\beta\|_{L^{1}(\Omega)} G_{\infty} m^{p} p}\left\{1-\lambda p m^{p}\|\alpha\|_{L^{1}(\Omega)} \liminf _{\xi \rightarrow+\infty} \frac{\max _{|t| \leq \xi}(-F(t))}{\xi^{p}}\right\}
$$

the problem $\left(P_{\lambda, \mu}^{f, g}\right)$ admits a sequence of solutions which are unbounded in $W_{0}^{1, p}(\Omega)$.

Proof. Our aim is to apply part (a) of Theorem 2.2. To this end, fix $\bar{\lambda} \in] \lambda_{1}, \lambda_{2}$ [ and let $g$ be a function that satisfies hypotheses $\left(g_{1}\right)-\left(g_{2}\right)$. Owing to $\bar{\lambda}<\lambda_{2}$, one has

$$
\mu_{g, \bar{\lambda}}:=\frac{1}{\|\beta\|_{L^{1}(\Omega)} G_{\infty} m^{p} p}\left\{1-\bar{\lambda} p m^{p}\|\alpha\|_{L^{1}(\Omega)} \liminf _{\xi \rightarrow+\infty} \frac{\max _{|t| \leq \xi \xi}(-F(t))}{\xi^{p}}\right\}>0 .
$$

Fix $0 \leq \bar{\mu}<\mu_{g, \pi}$ and denote by $X$ the Banach space $W_{0}^{1, p}(\Omega)$ endowed of the norm

$$
\|u\|:=\left(\int_{\Omega}|\nabla u(x)|^{p} d x\right)^{1 / p}
$$

Assume $j$ identically zero in $X$ and let $\Phi, \Psi: X \rightarrow \mathbb{R}$ defined as follows:

$$
\Phi(u):=\frac{\|u\|^{p}}{p}, \quad \Psi(u):=\int_{\Omega} H_{\bar{\lambda}}(u(x)) d x, \quad \forall u \in X,
$$


where

$$
H_{\bar{\lambda}}(u):=-\alpha(x) F(u)-\frac{\bar{\mu}}{\bar{\lambda}} \beta(x) G(u),
$$

for every $u \in X$.

By standard arguments, one has that $\Phi$ is Gâteaux differentiable and sequentially weakly lower semicontinuous and its Gâteaux derivative (at the point $u$ ) is the functional $\Phi^{\prime}(u) \in X^{*}$ given by

$$
\Phi^{\prime}(u)(v)=\int_{\Omega}|\nabla u(x)|^{p-2} \nabla u(x) \cdot \nabla v(x) d x
$$

for every $v \in X$. Furthermore, $\Psi$ is, in particular, sequentially weakly upper semicontinuous. Put $J_{\bar{\lambda}}(u):=\Phi(u)-\bar{\lambda} \Psi(u)$ for every $u \in X$. We want to prove that, under our hypotheses, there exists a sequence $\left\{\bar{u}_{n}\right\} \subset X$ of critical points for the functional $J_{\bar{\lambda}}$, that is, every element $\bar{u}_{n}$ satisfies

$$
J_{\bar{\lambda}}^{\circ}\left(\bar{u}_{n}, v-\bar{u}_{n}\right) \geq 0
$$

for every $v \in X$.

First of all, we will show that $\bar{\lambda}<1 / \gamma$. Hence, let $\left\{c_{n}\right\}$ be a real sequence such that $\lim _{n \rightarrow \infty} c_{n}=+\infty$ and

$$
\lim _{n \rightarrow \infty} \frac{\max _{|t| \leq c_{n}}(-F(t))}{c_{n}^{p}}=A
$$

Put $r_{n}=c_{n}^{p} / m^{p} p$ for every $n \in \mathbb{N}$. Taking into account formula (2.14), one has $\max _{x \in \bar{\Omega}}|v(x)| \leq$ $c_{n}$ for all $v \in X$ such that $\|v\|^{p}<p r_{n}$. Moreover, for every $n \in \mathbb{N}$, it follows that

$$
\begin{aligned}
\varphi\left(r_{n}\right) & =\inf _{\|u\|^{p}<p r_{n}} \frac{\sup _{\|v\|^{p}<p r_{n}} \int_{\Omega} H_{\bar{\lambda}}(v(x)) d x-\int_{\Omega} H_{\bar{\lambda}}(u(x)) d x}{r_{n}-\|u\|^{p} / p} \leq \frac{\sup _{\|v\|^{p}<p r_{n}} \int_{\Omega} H_{\bar{\lambda}}(v(x)) d x}{r_{n}} \\
& \leq \frac{\sup _{\|v\|^{p}<p r_{n}} \int_{\Omega}-\alpha(x) F(v(x)) d x}{r_{n}}+\frac{\bar{\mu}}{\bar{\lambda}} \frac{\sup _{\|v\|^{p}<p r_{n}} \int_{\Omega}-\beta(x) G(v(x)) d x}{r_{n}} \\
& \leq m^{p} p\|\alpha\|_{L^{1}(\Omega)} \frac{\max _{|t| \leq c_{n}}(-F(t))}{c_{n}^{p}}+\frac{\bar{\mu} m^{p} p}{\bar{\lambda}}\|\beta\|_{L^{1}(\Omega)} \frac{\max _{|t| \leq c_{n}}(-G(t))}{c_{n}^{p}} .
\end{aligned}
$$

Therefore, from the assumption $\left(f_{2}\right)$, one has $A<+\infty$. Hence, from hypothesis $\left(g_{2}\right)$, we obtain

$$
\lim _{n \rightarrow \infty} \frac{\max _{|t| \leq c_{n}}(-G(t))}{c_{n}^{p}}=G_{\infty} .
$$


Then,

$$
r \leq \liminf _{n \rightarrow+\infty} \varphi\left(r_{n}\right) \leq m^{p} p A\|\alpha\|_{L^{1}(\Omega)}+\frac{\bar{\mu} m^{p} p}{\bar{\lambda}}\|\beta\|_{L^{1}(\Omega)} G_{\infty}<+\infty
$$

Assumption $0<\bar{\mu}<\mu_{g, \bar{\lambda}}$ immediately yields

$$
r \leq m^{p} p\|\alpha\|_{L^{1}(\Omega)} A+\frac{\bar{\mu} m^{p} p}{\bar{\jmath}}\|\beta\|_{L^{1}(\Omega)} G_{\infty}<m^{p} p\|\alpha\|_{L^{1}(\Omega)} A+\frac{1-\bar{\lambda} p m^{p}\|\alpha\|_{L^{1}(\Omega)} A}{\bar{\lambda}} .
$$

Hence,

$$
\bar{\lambda}=\frac{1}{m^{p} p\|\alpha\|_{L^{1}(\Omega)} A+\left(1-\bar{\lambda} p m^{p}\|\alpha\|_{L^{1}(\Omega)} A\right) / \bar{\lambda}}<\frac{1}{\gamma} .
$$

We claim that the functional $J_{\bar{\lambda}}$ is unbounded from below. Let $\left\{\zeta_{n}\right\}$ be a real sequence such that $\lim _{n \rightarrow \infty} \zeta_{n}=+\infty$ and

$$
\lim _{n \rightarrow \infty} \frac{\left(-F\left(\zeta_{n}\right)\right)}{\zeta_{n}^{p}}=B
$$

For each $n \in \mathbb{N}$, define

$$
w_{n}(x):= \begin{cases}0, & \text { if } x \in \Omega \backslash B\left(\mathrm{x}_{0}, \tau\right), \\ \frac{\zeta_{n}}{\tau(1-\bar{\eta})}\left(\tau-\left|x-x_{0}\right|\right), & \text { if } x \in B\left(\mathrm{x}_{0}, \tau\right) \backslash B\left(\mathrm{x}_{0}, \bar{\eta} \tau\right), \\ \zeta_{n,} & \text { if } x \in B\left(\mathrm{x}_{0}, \bar{\eta} \tau\right) .\end{cases}
$$

One can prove that

$$
\left\|w_{n}\right\|^{p}=\int_{\Omega}\left|\nabla w_{n}(x)\right|^{p} d x=\zeta_{n}^{p} \omega_{\tau}\left[\frac{1-\bar{\eta}^{N}}{\tau^{p}(1-\bar{\eta})^{p}}\right] .
$$

Indeed,

$$
\begin{aligned}
\int_{\Omega}\left|\nabla w_{n}(x)\right|^{p} d x & =\int_{B\left(x_{0}, \tau\right) \backslash B\left(x_{0}, \bar{\eta} \tau\right)} \frac{\zeta_{n}^{p}}{\tau^{p}(1-\bar{\eta})^{p}} d x \\
& =\frac{\zeta_{n}^{p}}{(1-\bar{\eta})^{p} \tau^{p}}\left(\operatorname{meas}\left(B\left(x_{0}, \bar{\eta}\right)\right)-\operatorname{meas}\left(B\left(x_{0}, \bar{\eta} \tau\right)\right)\right) \\
& =\frac{\zeta_{n}^{p} \omega_{\tau}}{(1-\bar{\eta})^{p} \tau^{p}}\left(1-\bar{\eta}^{N}\right) .
\end{aligned}
$$


At this point, bearing in mind $\left(f_{1}\right)$, we infer

$$
\int_{\Omega}\left(-\alpha(x) F\left(w_{n}(x)\right)\right) d x \geq \int_{B\left(x_{0}, \bar{\eta} \tau\right)}\left(-\alpha(x) F\left(\zeta_{n}\right)\right) d x \geq-F\left(\zeta_{n}\right)\|\alpha\|_{L^{1}\left(B\left(x_{0}, \bar{\eta} \tau\right)\right)}, \quad \forall n \in \mathbb{N},
$$

and, clearly, one has

$$
-\bar{\lambda} \int_{\Omega}\left(-\alpha(x) F\left(w_{n}(x)\right)\right) d x \leq \bar{\lambda} F\left(\zeta_{n}\right)\|\alpha\|_{L^{1}\left(B\left(x_{0}, \bar{\eta} \tau\right)\right)}
$$

Moreover, owing to $\left(g_{1}\right)$, it follows that

$$
-\bar{\mu} \int_{\Omega}\left(-\beta(x) G\left(w_{n}(x)\right)\right) d x \leq 0
$$

for every $n \in \mathbb{N}$.

Hence, inequalities (3.20) and (3.21) imply that

$$
\begin{aligned}
J_{\bar{\lambda}}\left(w_{n}\right) & =\Phi\left(w_{n}\right)-\bar{\lambda} \Psi\left(w_{n}\right)=\frac{\left\|w_{n}\right\|^{p}}{p}-\bar{\lambda} \int_{\Omega}\left(-\alpha(x) F\left(w_{n}(x)\right)\right) d x-\bar{\mu} \int_{\Omega}\left(-\beta(x) G\left(w_{n}(x)\right)\right) d x \\
& \leq \frac{\left\|w_{n}\right\|^{p}}{p}-\bar{\lambda} \int_{\Omega}\left(-\alpha(x) F\left(w_{n}(x)\right)\right) d x \leq \frac{\zeta_{n}^{p} \omega_{\tau}}{p}\left[\frac{1-\bar{\eta}^{N}}{\tau^{p}(1-\bar{\eta})^{p}}\right]+\bar{\lambda} F\left(\zeta_{n}\right)\|\alpha\|_{L^{1}\left(B\left(x_{0}, \bar{\eta} \tau\right)\right)}
\end{aligned}
$$

for every $n \in \mathbb{N}$.

If $B<+\infty$, let $\epsilon \in]\left(\omega_{\tau} \bar{\eta}^{N} \sigma(N, p)\right) /\left(\bar{\lambda} p \tau^{p}\|\alpha\|_{L^{1}\left(B\left(x_{0}, \bar{\eta} \tau\right)\right)} B\right), 1\left[\right.$. By (3.15), there exists $v_{\epsilon}$ such that

$$
F\left(\zeta_{n}\right)<-\epsilon B \zeta_{n}^{p}, \quad \forall n>v_{\epsilon}
$$

Moreover,

$$
\begin{aligned}
J_{\bar{\lambda}}\left(w_{n}\right) & \leq \frac{\zeta_{n}^{p} \omega_{\tau}}{p}\left[\frac{1-\bar{\eta}^{N}}{\tau^{p}(1-\bar{\eta})^{p}}\right]-\bar{\lambda}\|\alpha\|_{L^{1}\left(B\left(x_{0}, \bar{\eta} \tau\right)\right)} \epsilon B \zeta_{n}^{p} \\
& =\zeta_{n}^{p}\left(\frac{\omega_{\tau} \bar{\eta}^{N} \sigma(N, p)}{p \tau^{p}}-\bar{\lambda}\|\alpha\|_{L^{1}\left(B\left(x_{0}, \bar{\eta} \tau\right)\right)} \epsilon B\right), \quad \forall n>v_{\epsilon} .
\end{aligned}
$$

Taking into account the choice of $\epsilon$, one has

$$
\lim _{n \rightarrow \infty} J_{\bar{\lambda}}\left(w_{n}\right)=-\infty
$$


If $B=+\infty$, let us consider

$$
M>\frac{\omega_{\tau} \bar{\eta}^{N} \sigma(N, p)}{\bar{\lambda} p \tau^{p}\|\alpha\|_{L^{1}\left(B\left(x_{0}, \bar{\eta} \tau\right)\right)}} .
$$

By (3.15), there exists $v_{M}$ such that

$$
F\left(\zeta_{n}\right)<-M \zeta_{n}^{p}, \quad \forall n>v_{M}
$$

Then,

$$
\begin{aligned}
J_{\bar{\lambda}}\left(w_{n}\right) & \leq \frac{\zeta_{n}^{p} \omega_{\tau}}{p}\left[\frac{1-\bar{\eta}^{N}}{\tau^{p}(1-\bar{\eta})^{p}}\right]-\bar{\lambda}\|\alpha\|_{L^{1}\left(B\left(x_{0}, \bar{\eta} \tau\right)\right)} M \zeta_{n}^{p} \\
& =\zeta_{n}^{p}\left(\frac{\omega_{\tau} \bar{\eta}^{N} \sigma(N, p)}{p \tau^{p}}-\bar{\lambda}\|\alpha\|_{L^{1}\left(B\left(x_{0}, \bar{\eta} \tau\right)\right)} M\right), \quad \forall n>v_{M} .
\end{aligned}
$$

Finally, inequality (3.26) implies that

$$
\lim _{n \rightarrow \infty} J_{\bar{\lambda}}\left(w_{n}\right)=-\infty
$$

From part (b) of Theorem 2.2, the functional $J_{\bar{\lambda}}$ admits a sequence of critical points $\left\{\bar{u}_{n}\right\} \subset X$ such that $\lim _{n \rightarrow \infty} \Phi\left(\bar{u}_{n}\right)=+\infty$. Since $\Phi$ is bounded on bounded sets and taking into account that $\lim _{n \rightarrow+\infty} \Phi\left(\bar{u}_{n}\right)=+\infty$, then $\left\{\bar{u}_{n}\right\}$ has to be unbounded. Moreover, if $\bar{u}_{n} \in X$ is a critical point of $J_{\bar{\lambda}}$, clearly, by definition, one has

$$
J_{\bar{\lambda}}^{\circ}\left(\bar{u}_{n}, v-\bar{u}_{n}\right) \geq 0
$$

for every $v \in X$.

We will prove that $\bar{u}_{n}$ solves problem $\left(P_{\lambda, \mu}^{f, g}\right)$. From (3.30), taking into account Proposition 2.1 and by the regularity of the term $\Phi$, one has

$$
\Phi^{\prime}\left(\bar{u}_{n}, v-\bar{u}_{n}\right)+\bar{\lambda}\left[-\Psi\left(\bar{u}_{n}, v-\bar{u}_{n}\right)\right]^{\circ} \geq 0
$$

for every $v \in X$.

From (3.31), it follows that

$$
\begin{gathered}
\int_{\Omega}\left|\nabla \bar{u}_{n}(x)\right|^{p-2} \nabla \bar{u}_{n}(x) \cdot \nabla\left(v(x)-\bar{u}_{n}(x)\right) d x+\bar{\lambda}\left[\int_{\Omega} \alpha(x) F\left(\bar{u}_{n}(x) ;\left(v(x)-\bar{u}_{n}(x)\right)\right) d x\right. \\
\left.+\frac{\bar{\mu}}{\bar{\lambda}} \int_{\Omega} \beta(x) G\left(\bar{u}_{n}(x) ;\left(v(x)-\bar{u}_{n}(x)\right)\right) d x\right]^{\circ} \geq 0, \quad \forall v \in X .
\end{gathered}
$$


By using Proposition 2.1 and from formula (2) on p. 77 in [24], we have

$$
\begin{aligned}
& {\left[\int_{\Omega} \alpha(x) F\left(\bar{u}_{n}(x) ;\left(v(x)-\bar{u}_{n}(x)\right)\right) d x+\frac{\bar{\mu}}{\bar{\lambda}} \int_{\Omega} \beta(x) G\left(\bar{u}_{n}(x) ;\left(v(x)-\bar{u}_{n}(x)\right)\right) d x\right]^{\circ}} \\
& \quad \leq \int_{\Omega} \alpha(x) F^{\circ}\left(\bar{u}_{n}(x) ;\left(v(x)-\bar{u}_{n}(x)\right)\right) d x+\frac{\bar{\mu}}{\bar{\lambda}} \int_{\Omega} \beta(x) G^{\circ}\left(\bar{u}_{n}(x) ;\left(v(x)-\bar{u}_{n}(x)\right)\right) d x .
\end{aligned}
$$

Then,

$$
\begin{aligned}
& \int_{\Omega}\left|\nabla \bar{u}_{n}(x)\right|^{p-2} \nabla \bar{u}_{n}(x) \cdot \nabla\left(v(x)-\bar{u}_{n}(x)\right) d x \\
& \quad+\bar{\lambda}\left[\int_{\Omega} \alpha(x) F^{\circ}\left(\bar{u}_{n}(x) ;\left(v(x)-\bar{u}_{n}(x)\right)\right) d x+\frac{\bar{\mu}}{\bar{\lambda}} \int_{\Omega} \beta(x) G^{\circ}\left(\bar{u}_{n}(x) ;\left(v(x)-\overline{\mathrm{u}}_{n}(x)\right)\right) d x\right] \geq 0,
\end{aligned}
$$

that is,

$$
\begin{aligned}
& -\int_{\Omega}\left|\nabla \bar{u}_{n}(x)\right|^{p-2} \nabla \bar{u}_{n}(x) \cdot \nabla\left(v(x)-\bar{u}_{n}(x)\right) d x \\
& \quad \leq \bar{\lambda} \int_{\Omega} \alpha(x) F^{\circ}\left(\bar{u}_{n}(x) ;\left(v(x)-\bar{u}_{n}(x)\right)\right) d x+\bar{\mu} \int_{\Omega} \beta(x) G^{\circ}\left(\bar{u}_{n}(x) ;\left(v(x)-\bar{u}_{n}(x)\right)\right) d x,
\end{aligned}
$$

for every $v \in X$. The proof is complete.

Remark 3.2. In [13], it is showed, under suitable assumptions on the potentials $F$ and $G$, the existence of infinitely many solutions for the following variational-hemivariational inequality problem. Find $u \in K$ fulfilling

$$
\begin{aligned}
& -\int_{\Omega}\left[|\nabla u(x)|^{p-2} \nabla u(x) \cdot \nabla(v(x)-u(x))+q(x)|u(x)|^{p-2} u(x)(v(x)-u(x))\right] d x \\
& \quad \leq \int_{\Omega}\left[\alpha(x) F^{\circ}(u(x) ;(v(x)-u(x)))+\beta(x) G^{\circ}(u(x) ;(v(x)-u(x)))\right] d x, \quad \forall v \in K,
\end{aligned}
$$

where $q \in L^{\infty}(\Omega)$ with ess $\inf _{x \in \Omega} q(x)>0$ and $K$ is a closed and convex set of the Sobolev space $W^{1, p}(\Omega)$ that contains the constant functions. Hence, roughly speaking, variationalhemivariational inequalities may be regarded as hemivariational inequalities subject to variational constraints. We pointed out that, in their treatment, the authors consider the presence of the constant functions. In our approach, for problem of Dirichlet type, the rule of the constant functions is played by the sequence $\left\{w_{n}\right\} \subset W_{0}^{1, p}(\Omega)$ defined in Theorem 3.1 
and previously introduced by Bonanno and Livrea in [29]. It is worth noticing that the techniques adopted here and introduced by Bonanno and Molica Bisci in [26] (see also [30]) are fundamentally proving Theorem 3.1, since the usual method utilized in order to obtain infinitely many solutions (see $[13,31]$ ) cannot be easily applied in the Dirichlet problems.

Remark 3.3. Assume that in Theorem 3.1, $f$ is a nonpositive continuous function. Hence, hypothesis $\left(f_{2}\right)$ can be written as follows:

$$
\left(f_{2}^{\prime}\right) \liminf _{\xi \rightarrow+\infty}\left((-F(\xi)) / \xi^{p}\right)<\kappa \lim \sup _{\xi \rightarrow+\infty}\left((-F(\xi)) / \xi^{p}\right),
$$

as well as

$$
\mu_{g, \lambda}:=\frac{1}{\|\beta\|_{L^{1}(\Omega)} G_{\infty} m^{p} p}\left\{1-\lambda p m^{p}\|\alpha\|_{L^{1}(\Omega)} \operatorname{meas}(\Omega) \liminf _{\xi \rightarrow+\infty} \frac{(-F(t))}{\xi^{p}}\right\} .
$$

Remark 3.4. If $f$ is such that condition $\left(f_{1}\right)$ holds and

$$
\liminf _{\xi \rightarrow+\infty} \frac{\max _{|t| \leq \xi}(-F(t))}{\xi^{p}}=0, \quad \limsup _{\xi \rightarrow+\infty} \frac{(-F(\xi))}{\xi^{p}}=+\infty
$$

clearly, hypothesis $\left(f_{2}\right)$ is verified and Theorem 3.1 guarantees the existence of infinitely many solutions for problem $\left(P_{\lambda, \mu}^{f, g}\right)$, for every pair $(\lambda, \mu) \in D$, where

$$
D:=] 0,+\infty\left[\times\left[0, \frac{1}{\|\beta\|_{L^{1}(\Omega)} G_{\infty} m^{p} p}[.\right.\right.
$$

Moreover, under the assumption $G_{\infty}=0$, the main result guarantees the existence of infinitely many solutions for problem $\left(P_{\lambda, \mu}^{f, g}\right)$, for every $\mu \geq 0$.

Remark 3.5. When $f, g: \mathbb{R} \rightarrow \mathbb{R}$ are continuous functions, the inequality $\left(P_{\lambda, \mu}^{f, g}\right)$ takes the form

$$
-\int_{\Omega}\left|\nabla u_{n}(x)\right|^{p-2} \nabla u(x) \cdot \nabla v(x) d x=\lambda \int_{\Omega} \alpha(x) f(u(x)) v(x) d x+\mu \int_{\Omega} \beta(x) g(u(x)) v(x) d x,
$$

for every $v \in W_{0}^{1, p}(\Omega)$.

Therefore, in such a case, a function $u \in W_{0}^{1, p}(\Omega)$ solves $\left(P_{\lambda, \mu}^{f, g}\right)$ if and only if it is a weak solution of the Dirichlet problem

$$
\begin{gathered}
\Delta p u=\lambda \alpha(x) f(u)+\mu \beta(x) g(u) \text { in } \Omega, \\
\left.u\right|_{\partial \Omega}=0 .
\end{gathered}
$$$$
\left(D_{\lambda, \mu}^{f, g}\right)
$$

Hence, Theorem 1.1 in Introduction is a particular case of Theorem 3.1 taking into account Remarks 3.3 and 3.4. 
We want to emphasize the fact that, replacing the condition at infinity of the potential $F$ by a similar one at zero, the existence of a sequence of pairwise distinct solutions (uniformly converging to zero) of problem $\left(P_{\lambda, \mu}^{f, g}\right)$, is achieved by using part (b) of Theorem 2.2 instead of part (a). For this goal, put

$$
\begin{array}{rlrl}
A^{*}:=\liminf _{\xi \rightarrow 0^{+}} \frac{\max _{|t| \leq \xi}(-F(t))}{\xi p}, & B^{*}:=\limsup _{\xi \rightarrow 0^{+}} \frac{(-F(\xi))}{\xi^{p}}, \\
\lambda_{1}^{*}:=\frac{\omega_{\tau} \bar{\eta}^{N} \sigma(N, p)}{p \tau^{p}\|\alpha\|_{L^{1}\left(B\left(x_{0}, \bar{\eta} \tau\right)\right)} B^{*}}, \quad \lambda_{2}^{*}:=\frac{1}{p m^{p}\|\alpha\|_{L^{1}(\Omega)} A^{*}} .
\end{array}
$$

Theorem 3.6. Let $f: \mathbb{R} \rightarrow \mathbb{R}$ be a locally essentially bounded function and put $F(\xi):=\int_{0}^{\xi} f(t) d t$, for every $\xi \in \mathbb{R}$. Assume that

$\left(f_{1}\right) \sup _{\xi \geq 0} F(\xi)=0$

$\left(f_{2}^{0}\right)$ and that

$$
\liminf _{\xi \rightarrow 0^{+}} \frac{\max _{|t| \leq \xi \xi}(-F(t))}{\xi^{p}}<\kappa \limsup _{\xi \rightarrow 0^{+}} \frac{(-F(\xi))}{\xi^{p}}
$$

where $\mathcal{\kappa}$ is given by (2.17).

Then, for every $\lambda \in] \lambda_{1}^{*}, \lambda_{2}^{*}[$, for every locally essentially bounded function $g: \mathbb{R} \rightarrow \mathbb{R}$ whose potential $G(\xi):=\int_{0}^{\xi} g(t) d t$ for every $\xi \in \mathbb{R}$, satisfies

$$
\begin{aligned}
& \left(g_{1}\right) \sup _{\xi \geq 0} G(\xi)=0, \\
& \left(g_{2}^{0}\right) G_{0}:=\lim _{\xi \rightarrow 0^{+}}\left(\max _{|t| \leq \xi}(-G(t)) / \xi^{p}\right)<+\infty,
\end{aligned}
$$

and for every $\mu \in\left[0, \mu_{g, \lambda}^{\star}[\right.$, where

$$
\mu_{g, \lambda}^{\star}:=\frac{1}{\|\beta\|_{L^{1}(\Omega)} G_{\infty} m^{p} p}\left\{1-\lambda p m^{p}\|\alpha\|_{L^{1}(\Omega)} \liminf _{\xi \rightarrow 0^{+}} \frac{\max _{|t| \leq \xi}(-F(t))}{\xi^{p}}\right\},
$$

the problem $\left(P_{\lambda, \mu}^{f, g}\right)$ possesses a sequence of nonzero solutions which strongly converge to 0 in $W^{1, p}(\Omega)$.

Proof. Taking $X, \Phi$, and $\Psi$ as in the proof of Theorem 3.1, fix $\bar{\lambda} \in] \lambda_{1}^{*}, \lambda_{2}^{*}[$, let $g$ be a function that satisfy hypotheses $\left(g_{1}\right)-\left(g_{2}^{0}\right)$ and take $0 \leq \bar{\mu}<\mu_{g, \lambda}^{\star}$. As first step, we will prove that $\bar{\lambda}<1 / \delta$. Then, let $\left\{c_{n}\right\}$ be a real sequence of positive numbers such that $\lim _{n \rightarrow \infty} c_{n}=0$ and

$$
\lim _{n \rightarrow \infty} \frac{\max _{|t| \leq c_{n}}(-F(t))}{c_{n}^{p}}=A^{*}
$$

Arguing in a similar way of Theorem 3.1, by using hypotheses $\left(f_{2}^{0}\right)$ and $\left(g_{2}^{0}\right)$, we can prove that

$$
\varphi\left(r_{n}\right) \leq m^{p} p\|\alpha\|_{L^{1}(\Omega)} \frac{\max _{|t| \leq c_{n}}(-F(t))}{c_{n}^{p}}+\frac{\bar{\mu} m^{p} p}{\bar{\lambda}}\|\beta\|_{L^{1}(\Omega)} \frac{\max _{|t| \leq c_{n}}(-G(t))}{c_{n}^{p}}
$$


for each $n \in \mathbb{N}$ and where $r_{n}=c_{n}^{p} / m^{p} p$. Since

$$
\delta \leq \liminf _{n \rightarrow \infty} \varphi\left(r_{n}\right) \leq m^{p} p A^{*}\|\alpha\|_{L^{1}(\Omega)}+\frac{\bar{\mu} m^{p} p}{\bar{\lambda}}\|\beta\|_{L^{1}(\Omega)} G_{0}<+\infty
$$

From $0<\bar{\mu}<\mu_{g, \bar{\lambda}^{\prime}}^{\star}$ the following inequalities hold

$$
\delta \leq m^{p} p\|\alpha\|_{L^{1}(\Omega)} A^{*}+\frac{\bar{\mu} m^{p} p}{\bar{\lambda}}\|\beta\|_{L^{1}(\Omega)} G_{0}<m^{p} p\|\alpha\|_{L^{1}(\Omega)} A^{*}+\frac{1-\bar{\lambda} p m^{p}\|\alpha\|_{L^{1}(\Omega)} A^{*}}{\bar{\lambda}} .
$$

Hence,

$$
\bar{\lambda}=\frac{1}{m^{p} p\|\alpha\|_{L^{1}(\Omega)} A^{*}+\left(1-\bar{\lambda} p m^{p}\|\alpha\|_{L^{1}(\Omega)} A^{*}\right) / \bar{\lambda}}<\frac{1}{\delta} .
$$

In the next step, we show that 0 is not a local minimum point for the functional $J_{\bar{\lambda}}$. Indeed, let $\left\{\zeta_{n}\right\}$ be a real sequence of positive numbers such that $\lim _{n \rightarrow \infty} \zeta_{n}=0$ and

$$
\lim _{n \rightarrow \infty} \frac{\left(-F\left(\zeta_{n}\right)\right)}{\zeta_{n}^{p}}=B^{*}
$$

Consider the sequence of functions $\left\{w_{n}\right\}$ defined in Theorem 3.1. Of course, $\left\{w_{n}\right\}$ strongly converges to zero in $X$, and from $\left(g_{1}\right)$ it follows that $J_{\bar{\lambda}}\left(w_{n}\right)<0$ for every integer $n$ sufficiently large. Since $J_{\bar{\lambda}}(0)=0$, this means that 0 is not a local minimum of $J_{\bar{\lambda}}$. Then, the unique global minimum of $\Phi$ is not a local minimum of the functional $J_{\bar{\lambda}}$. From part (b) of Theorem 2.2, we obtain a sequence $\left\{v_{n}\right\} \subset X$ of critical points of $J_{\bar{\lambda}}$ such that $\lim _{n \rightarrow \infty}\left\|v_{n}\right\|=0$. Moreover, again as in the proof of Theorem 3.1, one can show that every critical point of the functional $J_{\bar{\lambda}}$ is also a solution of problem $\left(P_{\lambda, \mu}^{f, g}\right)$. Thus, the proof is complete.

\section{Applications}

Finally, by using our results, we show the existence of infinitely many solutions in two concrete cases.

Example 4.1. Set

$$
a_{n}:=\frac{2 n !(n+2) !-1}{4(n+1) !}, \quad b_{n}:=\frac{2 n !(n+2) !+1}{4(n+1) !},
$$

for every $n \in \mathbb{N}$. 
Define the nonpositive (and discontinuous) function $h: \mathbb{R} \rightarrow \mathbb{R}$ as follows:

$$
h(\xi):= \begin{cases}-2(n+1) !\left[n^{p-1}(n+1) !^{p}-(n-1)^{p-1} n !^{p}\right], & \text { if } \left.\xi \in \bigcup_{n \geq 0}\right] a_{n}, b_{n}[ \\ 0, & \text { if } \xi \in \mathbb{R} \backslash\left(\bigcup_{n \geq 0}\right] a_{n}, b_{n}[) .\end{cases}
$$

Direct computations ensure that

$$
\limsup _{\xi \rightarrow+\infty} \frac{(-H(\xi))}{\xi^{p}}=+\infty, \quad \liminf _{\xi \rightarrow+\infty} \frac{(-H(\xi))}{\xi^{p}}=0
$$

where $H(\xi):=\int_{0}^{\xi} h(t) d t$, for every $\xi \in \mathbb{R}$.

Then, for every $(\lambda, \mu) \in] 0,+\infty[\times[0,+\infty$ [ and for every nonpositive and locally essentially bounded function $g: \mathbb{R} \rightarrow \mathbb{R}$ with potential $G$ satisfying

$$
\lim _{\xi \rightarrow+\infty} \frac{G(\xi)}{\xi^{p}}=0
$$

the following problem $\left(P_{\lambda, \mu}^{h, g}\right)$.

Find $u \in W_{0}^{1, p}(\Omega)$ fulfilling

$$
\begin{aligned}
& -\int_{\Omega}|\nabla u(x)|^{p-2} \nabla u(x) \cdot \nabla(v(x)-u(x)) d x \\
& \quad \leq \lambda \int_{\Omega} H^{\circ}(u(x) ;(v(x)-u(x))) d x+\mu \int_{\Omega} G^{\circ}(u(x) ;(v(x)-u(x))) d x,
\end{aligned}
$$

for every $v \in W_{0}^{1, p}(\Omega)$, possesses a sequence of solutions which are unbounded in $W_{0}^{1, p}(\Omega)$.

The next is a simple consequence of Theorem 1.1.

Example 4.2. Define the nonpositive continuous function $f: \mathbb{R} \rightarrow \mathbb{R}$ as follows:

$$
f(t):= \begin{cases}-t \cos ^{2}(\ln (t)), & \text { if } t>0 \\ 0, & \text { if } t \leq 0\end{cases}
$$

Since

$$
\begin{aligned}
\liminf _{\xi \rightarrow+\infty} \frac{\int_{0}^{\xi}(-f(t)) d t}{\xi^{2}}=\frac{2-\sqrt{2}}{8} \\
\limsup _{\xi \rightarrow+\infty} \frac{\int_{0}^{\xi}(-f(t)) d t}{\xi^{2}}=\frac{2+\sqrt{2}}{8}
\end{aligned}
$$


one has

$$
\liminf _{\xi \rightarrow+\infty} \frac{\int_{0}^{\xi}(-f(t)) d t}{\xi^{2}}<\frac{1}{4} \limsup _{\xi \rightarrow+\infty} \frac{\int_{0}^{\xi}(-f(t)) d t}{\xi^{2}} .
$$

From Theorem 1.1, for each

$$
\lambda \in \Lambda:=] \frac{64}{2+\sqrt{2}}, \frac{16}{2-\sqrt{2}}[
$$

for every nonpositive continuous function $g: \mathbb{R} \rightarrow \mathbb{R}$ such that $\left(g_{1}^{\prime}\right)$ holds and for every $\mu \in\left[0, \widehat{\mu}_{g, \lambda}[\right.$, where

$$
\widehat{\mu}_{g, \lambda}:=\frac{\lambda(2-\sqrt{2})-16}{8}\left(\lim _{\xi \rightarrow+\infty} \frac{\int_{0}^{\xi} g(t) d t}{\xi^{2}}\right)^{-1},
$$

the following problem

$$
\begin{gathered}
\left.-u^{\prime \prime}=\lambda f(u)+\mu g(u) \text { in }\right] 0,1[, \\
u(0)=u(1)=0,
\end{gathered}
$$

possesses a sequence of weak solutions which are unbounded in $W_{0}^{1,2}(] 0,1[)$.

For instance, for each $(\lambda, \mu) \in \Lambda \times[0,+\infty$, the Dirichlet problem

$$
\begin{gathered}
\left.u^{\prime \prime}+\mu \sqrt{|u|}=\lambda f(u) \quad \text { in }\right] 0,1[ \\
u(0)=u(1)=0,
\end{gathered}
$$

possesses a sequence of pairwise distinct positive classical solutions.

\section{Acknowledgment}

The authors warmly thank professor G. Bonanno for many stimulating conversations on the subject treated in this paper.

\section{References}

[1] P. D. Panagiotopoulos, Hemivariational Inequalities, Springer, New York, NY, USA, 1993, Applications in mechanics and engineerin.

[2] P. D. Panagiotopoulos, Inequality Problems in Mechanics and Applications, Birkhäuser, Boston, Mass, USA, 1985, Convex and Nonconvex Energy Function.

[3] Z. Naniewicz and P. D. Panagiotopoulos, Mathematical Theory of Hemivariational Inequalities and Applications, vol. 188, Marcel Dekker, New York, NY, USA, 1995. 
[4] D. Motreanu and P. D. Panagiotopoulos, Minimax Theorems and Qualitative Properties of the Solutions of Hemivariational Inequalities, vol. 29 of Nonconvex Optimization and its Applications, Kluwer Academic, Dordrecht, The Netherlands, 1999.

[5] D. Motreanu and V. Rădulescu, "Existence results for inequality problems with lack of convexity," Numerical Functional Analysis and Optimization, vol. 21, no. 7-8, pp. 869-884, 2000.

[6] D. Motreanu and V. Rădulescu, Variational and Non-Variational Methods in Nonlinear Analysis and Boundary Value Problems, vol. 67 of Nonconvex Optimization and its Applications, Kluwer Academic, Boston, Mass, USA, 2003.

[7] V. Rădulescu, Analyse de Quelques Problèmes aux Limites Elliptiques Non Linéaires, Habilitation à Diriger des Recherches, Université Pierre et Marie Curie, Paris, France, 2003.

[8] V. D. Rădulescu, Qualitative Analysis of Nonlinear Elliptic Partial Differential Equations: Monotonicity, Analytic, and Variational Methods, vol. 6 of Contemporary Mathematics and Its Applications, Hindawi Publishing Corporation, New York, NY, USA, 2008.

[9] G. Bonanno and P. Candito, "On a class of nonlinear variational-hemivariational inequalities," Applicable Analysis, vol. 83, no. 12, pp. 1229-1244, 2004.

[10] N. Costea and V. Rădulescu, "Existence results for hemivariational inequalities involving relaxed $\eta-\alpha$ monotone mappings," Communications in Applied Analysis, vol. 13, no. 3, pp. 293-303, 2009.

[11] G. D’Aguì and D. O’Regan, "Infinitely many solutions for a class of elliptic variationalhemivariational inequality problems," Studia Universitatis Babeş-Bolyai. Mathematica, vol. 55, no. 4, pp. 73-82, 2010.

[12] S. A. Marano, G. Molica Bisci, and D. Motreanu, "Multiple solutions for a class of elliptic hemivariational inequalities," Journal of Mathematical Analysis and Applications, vol. 337, no. 1, pp. 85-97, 2008

[13] S. A. Marano and D. Motreanu, "Infinitely many critical points of non-differentiable functions and applications to a Neumann-type problem involving the $p$-Laplacian," Journal of Differential Equations, vol. 182, no. 1, pp. 108-120, 2002.

[14] S. A. Marano and N. S. Papageorgiou, "On some elliptic hemivariational and variationalhemivariational inequalities," Nonlinear Analysis. Theory, Methods E Applications, vol. 62, no. 4, pp. 757-774, 2005.

[15] S. A. Marano and D. Motreanu, "On a three critical points theorem for non-differentiable functions and applications to nonlinear boundary value problems," Nonlinear Analysis. Theory, Methods $\mathcal{E}$ Applications, vol. 48, pp. 37-52, 2002.

[16] G. Bonanno and G. Molica Bisci, "Infinitely many solutions for a Dirichlet problem involving the p-Laplacian," Proceedings of the Royal Society of Edinburgh. Section A, vol. 140, no. 4, pp. 737-752, 2010.

[17] G. Bonanno, G. Molica Bisci, and D. O’Regan, "Infinitely many weak solutions for a class of quasilinear elliptic systems," Mathematical and Computer Modelling, vol. 52, no. 1-2, pp. 152-160, 2010.

[18] F. Obersnel and P. Omari, "Positive solutions of elliptic problems with locally oscillating nonlinearities," Journal of Mathematical Analysis and Applications, vol. 323, no. 2, pp. 913-929, 2006.

[19] P. Omari and F. Zanolin, "An elliptic problem with arbitrarily small positive solutions," in Proceedings of the Conference on Nonlinear Differential Equations, vol. 5 of Electronic Journal of Differential Equations: Conference, pp. 301-308, Southwest Texas State University, San Marcos, Tex, USA.

[20] P. Omari and F. Zanolin, "Infinitely many solutions of a quasilinear elliptic problem with an oscillatory potential," Communications in Partial Differential Equations, vol. 21, no. 5-6, pp. 721-733, 1996.

[21] G. Bonanno and A. Chinnì, "Existence of three solutions for a perturbed two-point boundary value problem," Applied Mathematics Letters, vol. 23, no. 7, pp. 807-811, 2010.

[22] G. Bonanno and G. D'Aguì, "Multiplicity results for a perturbed elliptic Neumann problem," Abstract and Applied Analysis, vol. 2010, Article ID 564363, pp. 1-10, 2010.

[23] G. Bonanno and G. Molica Bisci, "A remark on perturbed elliptyic Neumann problems," Studia Universitatis Babeş-Bolyai. Mathematica, vol. 55, no. 4, pp. 17-25, 2010.

[24] F. H. Clarke, Optimization and Nonsmooth Analysis, vol. 5 of Classics in Applied Mathematics, SIAM, Philadelphia, Pa, USA, 2nd edition, 1990.

[25] K. C. Chang, "Variational methods for nondifferentiable functionals and their applications to partial differential equations," Journal of Mathematical Analysis and Applications, vol. 80, no. 1, pp. 102-129, 1981.

[26] G. Bonanno and G. Molica Bisci, "Infinitely many solutions for a boundary value problem with discontinuous nonlinearities," Boundary Value Problems, vol. 2009, Article ID 670675, pp. 1-20, 2009. 
[27] B. Ricceri, "A general variational principle and some of its applications," Journal of Computational and Applied Mathematics, vol. 113, no. 1-2, pp. 401-410, 2000.

[28] G. Talenti, "Some inequalities of Sobolev type on two-dimensional spheres," in General inequalities, W. Walter, Ed., vol. 80 of International Series of Numerical Mathematics, pp. 401-408, Birkhäuser, Basel, Switzerland, 1987.

[29] G. Bonanno and R. Livrea, "Multiplicity theorems for the Dirichlet problem involving the $p$ Laplacian," Nonlinear Analysis. Theory, Methods \& Applications, vol. 54, no. 1, pp. 1-7, 2003.

[30] G. Bonanno and G. D'Aguì, "On the Neumann problem for elliptic equations involving the $p$ Laplacian," Journal of Mathematical Analysis and Applications, vol. 358, no. 2, pp. 223-228, 2009.

[31] B. Ricceri, "Infinitely many solutions of the Neumann problem for elliptic equations involving the p-Laplacian," The Bulletin of the London Mathematical Society, vol. 33, no. 3, pp. 331-340, 2001. 